\title{
SMR
}

\section{A putative miR172-targeted CeAPETALA2-like gene is involved in floral patterning regulation of the orchid Cymbidium ensifolium}

\author{
F.X. Yang, G.F. Zhu, Z. Wang, H.L. Liu and D. Huang \\ Guangdong Key Laboratory of Ornamental Plant Germplasm Innovation and \\ Utilization, Environmental Horticulture Research Institute, \\ Guangdong Academy of Agricultural Sciences, Guangzhou, China \\ Corresponding author: G.F. Zhu \\ E-mail: genfazhu@163.com
}

Genet. Mol. Res. 14 (4): 12049-12061 (2015)

Received January 30, 2015

Accepted May 18, 2015

Published October 5, 2015

DOI http://dx.doi.org/10.4238/2015.October.5.18

\begin{abstract}
APETALA2 plays critical roles in establishing meristem and organ identity during plant floral development. In this study, we obtained a CeAP2-like gene by using the mRNA differential display technique to analyze the wild type and a multitepal mutant of the orchid Cymbidium ensifolium. The fulllength cDNA encoding the CeAP2-like transcription factor shows significant similarity to the cDNA of $A P 2$ from Erycina pusilla and contains nucleotides complementary to miR 172 . Using a transient gene expression system of Arabidopsis protoplasts, we found that the accumulation of CeAP2-like protein and transcripts was negatively regulated by miR172, indicating this gene as a putative target of miR172. Northern blotting revealed that CeAP2-like is dominantly
\end{abstract}


expressed in the sepals and petals of the wild-type flower, and shows low expression in the gynostemium. In contrast, the accumulation of CeAP2-like transcripts decreased significantly, especially in the central part of the mutant flower, corresponding to its abnormal petals and the absence of the gynostemium. Furthermore, we found an antagonistic expression pattern between CeAP2-like and $A G A M O U S$ in the wild type, representing A- and C-class genes that specify floral organ fate. However, this antagonistic distribution was modified in the multitepal mutant, and both genes showed lower expression than that in the wild type. This result suggested that the balance between CeAP2-like and AGAMOUS activity was important for the regulation of floral patterning in $C$. ensifolium. This study represents the first report on a class A gene and its regulatory role for floral development in the orchid C. ensifolium.

Key words: Orchid; Cymbidium ensifolium; Floral patterning; APETALA2-like; miR172; AGAMOUS

\section{INTRODUCTION}

Orchids possess highly specialized floral organs, including three sepals in whorl 1, two petals and one lip in whorl 2, and a gynostemium in the center of the flower (Wang et al., 2011). The lip is a highly evolved petal, the shape of which is ovate to triangular, and it often possesses a kinked to undulate margin; the gynostemium is fused by the male and female reproductive organs and contains four pollinia on a semicircular viscidium (Schiestl and Cozzolino, 2008) (Figure 1A and 1B). These unique floral morphological synapomorphies define an unusually coherent group among monocots, representing a highly advanced and terminal line of floral evolution that is still actively evolving (Dressler, 1993). The orchid family thus experiences high speciation rates and contains approximately 750 genera with over 25,000 species (Dressler, 2005). In association with the enormous number of orchid species, extraordinary floral diversity is also observed in the family, which has drawn great horticultural interest (Ghazoul, 2006; Soltis and Soltis, 2014).

Despite the unique developmental reproductive biology of orchids and their horticultural and economic value, the molecular bases of floral pattern regulation and floral diversity in these species remain relatively unexplored, in comparison with those of other species-rich plant families (Eveland et al., 2014; Teo et al., 2014). According to the floral quartet model, floral morphogenesis is controlled by many transcription factors, including $\mathrm{ABCDE}$ homeotic genes (Causier et al., 2010). Among these genes, the A-class genes APETALA1 (AP1) and AP2 confer sepal identity in the first floral whorl (Keck et al., 2003). Their activity overlaps with that of the B-class genes AP3 and PISTILLATA (PI) in the second whorl, which cause petal development (Pelaz et al., 2000). AP3, PI, and the C-class gene AGAMOUS (AG) specify stamen identity in whorl 3, while $A G$ in whorl 4 promotes carpel development (Theissen and Becker, 2004; Melzer and Theissen, 2009). D class genes are related to ovule identity, and E class genes are broadly expressed in all four whorls and 
correlate to the floral meristem establishment (Melzer et al., 2009). Moreover, studies in Arabidopsis thaliana have shown that $A P 2$ and $A G$ (A- and C-class genes, respectively) act antagonistically to specify perianth and reproductive organs (Bowman et al., 1989, 1991; Ó’Maoiléidigh et al., 2014).

Recent studies on floral development-related genes in Phalaenopsis orchids have indicated that several MADS-box transcription factors act as ABCDE demotic genes after cloning, expression, and functional analysis (Tsai and Chen, 2006; Su et al., 2013). For example, expression studies have shown that the identity of the petals and lip in whorl 2 is specified by the combinatorial interaction of four different DEFICIENS-like MADSbox genes, which were generated from B-class gene duplication (Hsiao et al., 2013), and the C- and D-class MADS-box genes from Phalaenopsis equestris (Orchidaceae) were reported to display functions in gynostemium and ovule development (Tsai et al., 2008; Chang et al., 2010). However, owing to lack of knowledge, extensive investigations are required to further understand the floral $\mathrm{ABCDE}$ model in orchid, as shown by Wang et al. (2011). Moreover, nearly all of the evidence regarding these ABCDE demotic genes has been derived from Phalaenopsis, and studies in other orchid genera are greatly limited (Huang et al., 2012; Yang et al., 2014).

In the present study, we studied the wild type and a multitepal mutant of Cymbidium ensifolium, which is an economically important species of flowering orchid cultivated in China, Japan, Korea, and Southeast Asia. Due to its beautiful and fragrant flowers, Cymbidium is highly desirable in traditional flower markets in China, and its floral traits have attracted significant horticultural attention (Wang et al., 2014). To identify the genes related to various floral traits in this species, we isolated genes that were differentially expressed between the wild type and the multitepal mutant using the mRNA display technique. A sequence homologous to $A P 2$ was thereby obtained. We cloned the full lengths of the open reading frame by rapid amplification of the $5^{\prime} \mathrm{cDNA}$ end (5'RACE) to characterize this orchid AP2-like gene. Similar to AP2 in A. thaliana, this gene contains sequence motifs complementary to miR172, the microRNA that has been shown to regulate $A P 2$ expression (Zhu and Helliwell, 2011).

By using transient gene expression system of Arabidopsis protoplasts, we examined the regulation of CeAP2-like by miR172. Our results from semi-quantitative reversetranscription polymerase chain reaction (RT-PCR) analysis of CeAP2-like expression and fluorescence signal of the CeAP2-like-GFP fusion protein confirmed that the accumulation of CeAP2-like protein and transcripts was negatively regulated by miR172, indicating this gene as a putative target of miR172 in C. ensifolium. In addition, this gene is expressed in various floral organs, and it shows modified expression in the mutant flower, which is consistent with the abnormal floral organs. Moreover, an increase in the expression of CeAP2-like and a decrease in the accumulation of $A G$ were detected in the outer whorls of wild-type flowers. Expression of both these genes was modified in the multitepal mutant, and both genes showed lower expression than in the wild type, which further suggested that the mutual antagonism of $C e A P 2$-like and $A G$ was involved in the regulation of orchid floral development. These results together indicated that CeAP2-like plays critical roles in the regulation of floral patterning in C. ensifolium. The present study increases current understanding of the molecular mechanisms of orchid gynostemium morphogenesis. 


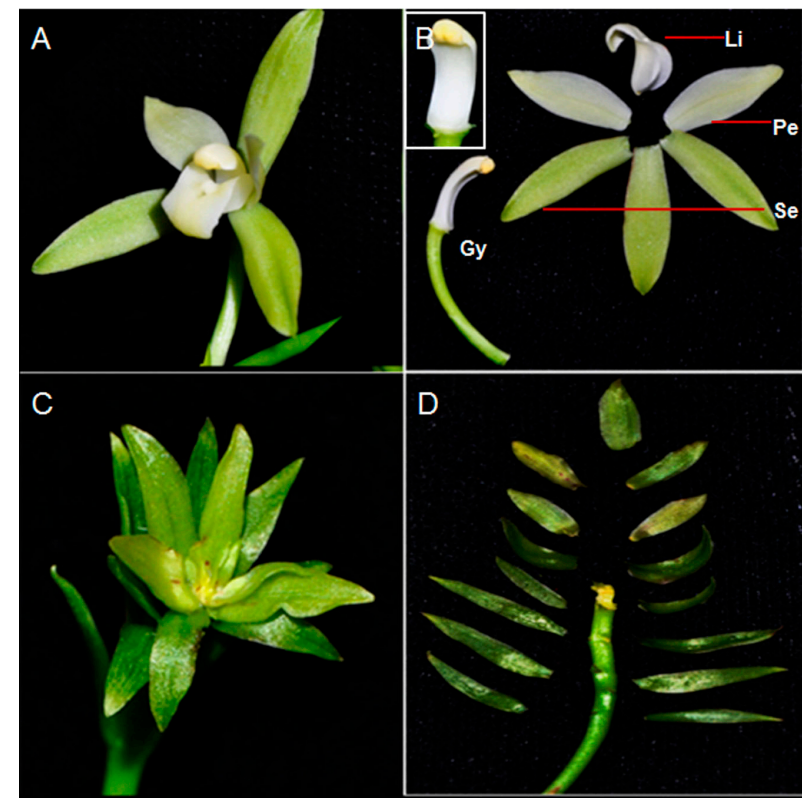

Figure 1. Floral morphogenesis of wild-type plant and multitepal mutant of Cymbidium ensifolium. A. B. Wildtype flower of $C$. ensifolium has three sepals and three petals. One petal is morphologically different in structure and is known as the labellum (or lip). The male and female reproductive parts are fused in the center of the flower, known as the gynostemium (or column). C. D. Flower of multitepal mutant exhibits petal-to-sepal transformations, generates more sepals, while the gynostemium is absent in the center of the flower. Se: sepals (whorl 1); Pe: petals and Li: lip (whorl 2); Gy: gynostemium (whorl 3 + whorl 4).

\section{MATERIAL AND METHODS}

\section{Plant materials and growth conditions}

The wild-type plants and multitepal mutants of $C$. ensifolium 'Tianesu' and 'Cuiyumudan' used in this study were collected from the cultivation base of the Floricultural Research Institute, Guangdong Academy of Agricultural Sciences, China. All of the plants were grown and maintained in pots in a greenhouse at day/night temperatures of $26^{\circ} / 22^{\circ} \mathrm{C}$ under a 16-h light/8-h dark photoperiod.

\section{Sequence alignment and phylogenetic analysis}

Full-length amino acid sequences were aligned by the Clustal X 1.83 program. The sequence alignment was further adjusted manually using the BioEdit software (http://www. mbio.ncsu.edu/bioedit/bioedit.html). The amino acid substitution model was calculated using Model Generator v0.84, and the optimal model of "JTT + G" was selected. Phylogenetic relationships were reconstructed using a maximum-likelihood method in the PHYML software with the JTT amino acid substitution model. 


\section{Northern blot analysis}

Total RNA extracted from different tissues of $C$. ensifolium using the Trizol ${ }^{\circledR}$ reagent (Life Technologies) was separated on 1.5\% formaldehyde-MOPS agarose gels and blotted onto $\mathrm{N}+$ membranes (Millipore Corporation, http://www.millipore.com). Hybridization was performed at $68^{\circ} \mathrm{C}$ in PerfectHybTM Plus Buffer (Sigma-Aldrich). Probes were labeled with 32P-dATP using the Klenow fragment (Takara).

For miR172 detection, small RNAs were detected following the electrophoresis of total RNA through a denaturing $15 \%$ polyacrylamide gel and transferred to cellulose acetate membranes (Millipore Corporation, http://www.millipore.com). Hybridization was performed at $35^{\circ} \mathrm{C}$ in PerfectHyb ${ }^{\mathrm{TM}}$ Plus Buffer (Sigma-Aldrich). Probes were labeled with $\left.{ }^{32} \mathrm{P}\right]$-dATP using terminal deoxynucleotidyl transferase (Takara). The intensity of the hybridized bands was assessed with a Bio-profile Bio 1D image analyzer (Vilber Lourmat, Marne La Vallée, France).

\section{GFP fusion and fluorescence analysis}

For GFP fusion construction, CeAP2-like-GFP and miR172 resistant $m$ CeAP2-like-GFP were driven by the $35 \mathrm{~S}$ promoter in the pBI221 vector. (Primers are shown in Table S1). The miR172-resistant version of $m C e A P 2$-like contained three silent mutations within the miR172complementary domain of the CeAP2-like genomic clone. It thus increased the number of mismatches between miR172 and $m C e A P 2$-like without altering the amino acid sequence [using the method as described by Liang et al. (2014)]. For the miRNA/AP2 coinfiltration experiments, equal volumes of plasmid DNA containing 35S:ath-MIR172a $(\mathrm{OD} 260 / 280=1.85)$ and 35S: CeAP2-like $(\mathrm{OD} 260 / 280=1.97)$ or 35S:mCeAP2-like $(\mathrm{OD} 260 / 280=2.14)$ were mixed before transformation into Arabidopsis protoplasts. Transient protein expression analysis in Arabidopsis protoplasts was performed as described by Abel and Theologis (1994). Fluorescence assays were conducted $12 \mathrm{~h}$ after transformation using a confocal laser-scanning microscope, as described elsewhere. Arabidopsis protoplasts transiently expressing 35S:ath-MIR172a/35S:CeAP2-like and 35S:ath-MIR172a/35S:mCeAP2-like were separately collected for RNA extraction.

\section{Quantitative reverse-transcription PCR (RT-PCR)}

Total RNA was extracted from Arabidopsis protoplasts using Trizol ${ }^{\circledR}$ reagent (Life Technologies) and treated with DNase I (Fermentas). The OneStep RT-PCR Kit (Fermentas) with $20 \mathrm{pM}$ primers was used to synthesize cDNA from $1 \mu \mathrm{g}$ RNA. PCRs were performed for 23 to 28 cycles, and ath-MIR172a was used as an internal standard. Each RT-PCR experiment was repeated at least three times.

\section{RESULTS}

\section{Isolation of the CeAP2-like gene in C. ensifolium}

Floral morphogenesis is one of the most attractive characteristics of Cymbidium, which has led to its cultivation and breeding. To identify genes associated with specific floral organ development, a multitepal mutant was employed in this study. The mutant exhibited petal-to-sepal transformations and more sepals overall (Figure 1C and D). Moreover, when the 
mutant was compared to the wild type, the gynostemium was absent in the mutant, suggesting interference with normal floral homeotic gene function in the perianth organs. We examined the transcriptional variations between wild-type and multitepal flowers using the mRNA differential display technique. A total of 89 differentially expressed fragments were identified, with 67 up-regulated and 22 down-regulated in the multitepal mutant.

Sequence analysis found that one of the fragments showed high homology to the cDNAs of AP2-9 from Erycina pusilla and AP2-like from Vitis vinifera, as well as to those of $A P 2$ from Musa acuminate and A. thaliana (Figure S1). We therefore designated the cDNA as CeAP2-like. Using rapid amplification of cDNA ends technology (5'RACE-PCR), we obtained the full 1449-nt length of the CeAP2-like cDNA sequence (Table S2).

The deduced CeAP2-like protein had 482 amino acid residues and possessed two AP2 domains, which are characteristic of transcriptional factors from the AP2 family. Seven DNAbinding sites, two protein-binding sites and a nuclear localization signal sequence were found in the CeAP2-like sequence (Figure 2A, Figure S2). Multiple sequence alignments with other APETALA2 proteins of plants demonstrated that CeAP2-like is very closely related to AP2-9 in E. pusilla with $87.6 \%$ identity (Figure 2B).
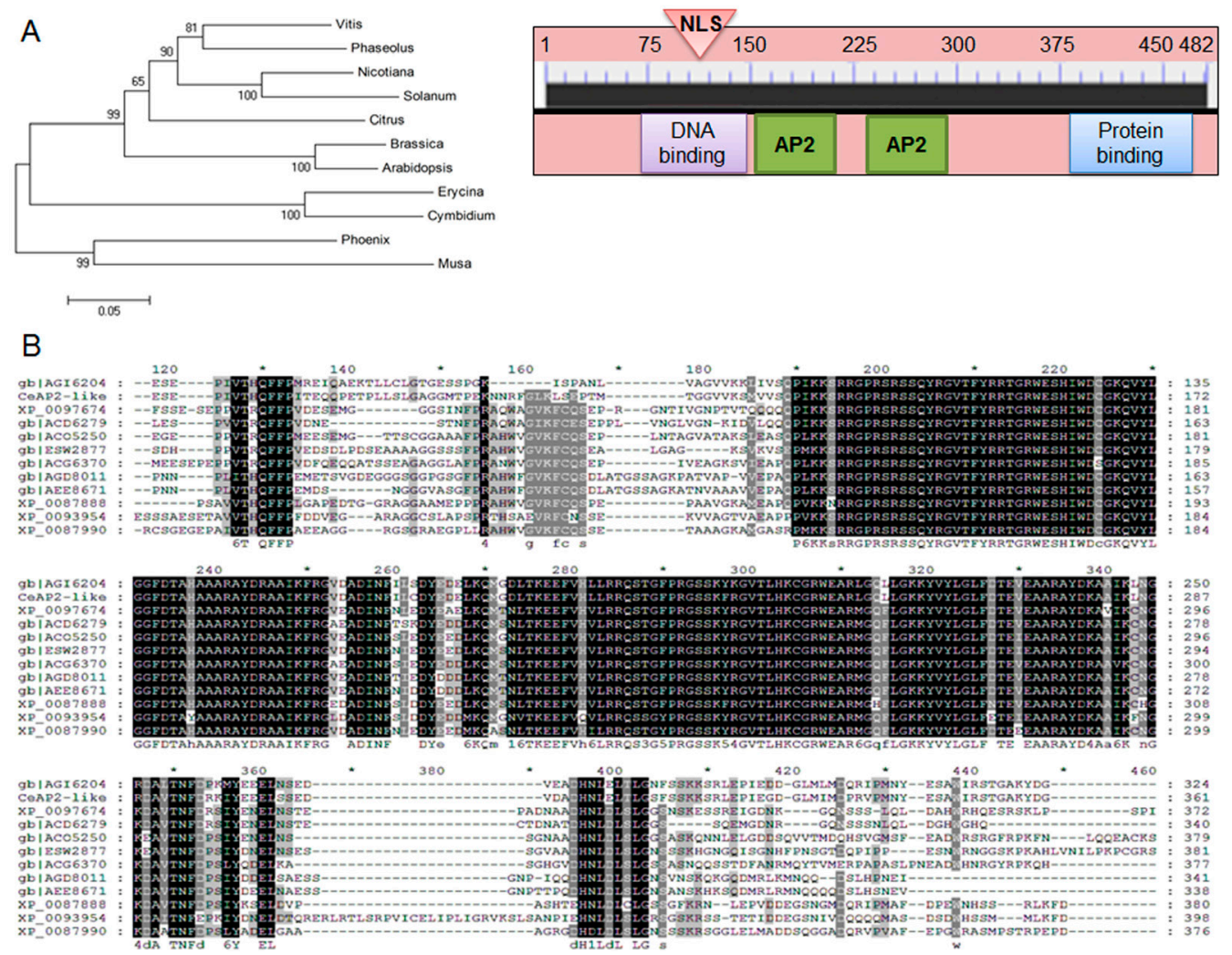

Figure 2. Phylogenic analysis between identified CeAP2-like sequence and its homologs in other species. A. Phylogenic analysis and the simplified protein structure of the identified CeAP2-like sequence. B. Alignment of the deduced amino acid sequences of CeAP2-like and other AP2-related proteins. The multiple alignments were generated by a maximum-likelihood method in the PHYML software with the JTT amino acid substitution model. 
Furthermore, in silico RNA hybridization revealed a sequence complementary to miR172 in the CeAP2-like cDNA, and the position of this sequence was similar to that in other miR172-targeted $A P 2$ genes from different plants (Figure 3). Since the regulation of the AP2domain genes by miR 172 has been broadly reported in both monocotyledons and dicotyledons (Zhu and Helliwell 2011; Varkonyi-Gasic et al., 2012), our findings indicated CeAP2-like as a putative target gene of miR172 in C. ensifolium.

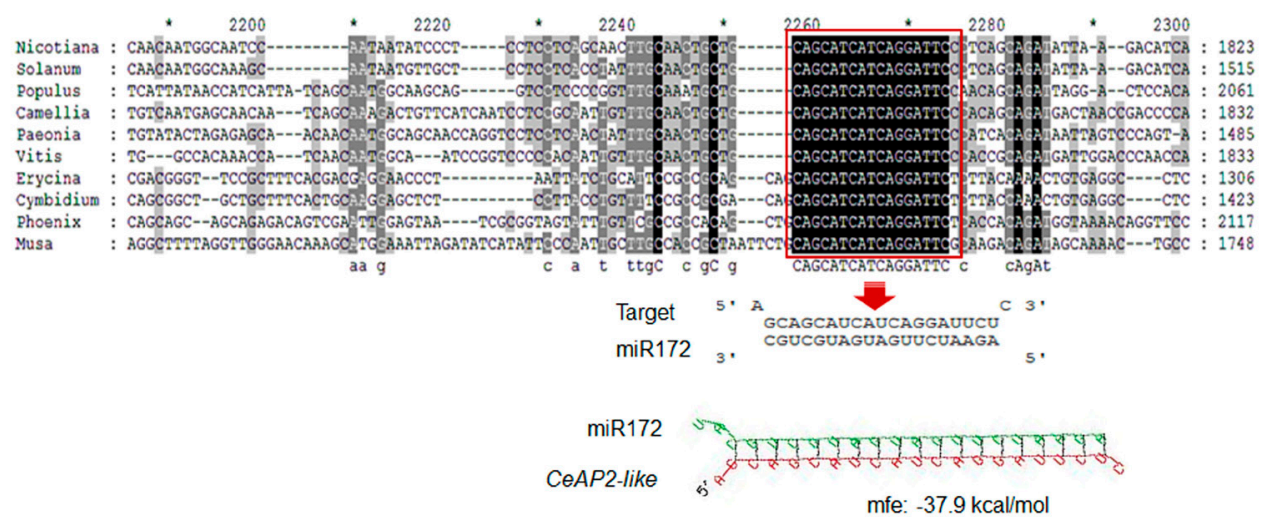

Figure 3. Sequence complementary to miR172 in the CeAP2-like gene. The comparison of complementary site of miR172 to CeAP2-like (indicated by the red frame). Free energy of duplex structures was calculated using the RNA hybrid software (http://bibiserv.techfak.uni-bielefeld.de/rnahybrid).

\section{Regulation of CeAP2-like expression by miR172}

To confirm the hypothesis that CeAP2-like could be targeted by miR172, we first detected the presence of miR172 in C. ensifolium by small RNA hybridization using an antisense probe for miR172a. Our results indicated that a small single-stranded RNA of $\sim 21 \mathrm{nt}$ accumulated in the floral organs, especially in the flower bud. This finding demonstrated the conservation of miR172 in C. ensifolium (Figure 4). To further determine whether a miR172/ $A P 2$ regulatory mode existed in $C$. ensifolium, we constructed a GFP-tagged CeAP2-like fusion and a miR172-resistant mCeAP2-like-GFP driven by 35S, respectively, and examined the fluorescence signals from Arabidopsis protoplasts transiently coexpressing miR172/CeAP2like-GFP or miR172/mCeAP2-like-GFP. In comparison with the fused protein containing the CeAP2-like sequence, the one containing the miR172-resistant mCeAP2-like sequence displayed a strong signal in the nucleus (Figure 5A). This confirmed that the translation of CeAP2-like was negatively regulated by miR172. Moreover, a weak signal was also detected in Arabidopsis protoplasts transiently coexpressing 35S:miR172/35S:CeAP2-like, suggesting that miR172 was insufficient to clear $A P 2$ mRNA, which was consistent with the results of previous reports in other species (Wollmann et al., 2010; Chung et al., 2010).

Meanwhile, Arabidopsis protoplasts from last step were collected, and the RNAs were extracted for semi-quantitative RT-PCR, to analyze the transcript abundance of CeAP2-like. In accordance with the fluorescence results from the GFP fusion experiments, the cleavage sitespanning fragment of $\mathrm{mCeAP2}$-like was expressed to a greater degree than that of CeAP2-like [method described by Yang et al. (2009) was used] (Figure 5B). This result further confirmed that miR172/AP2 regulation was conserved in C. ensifolium. 


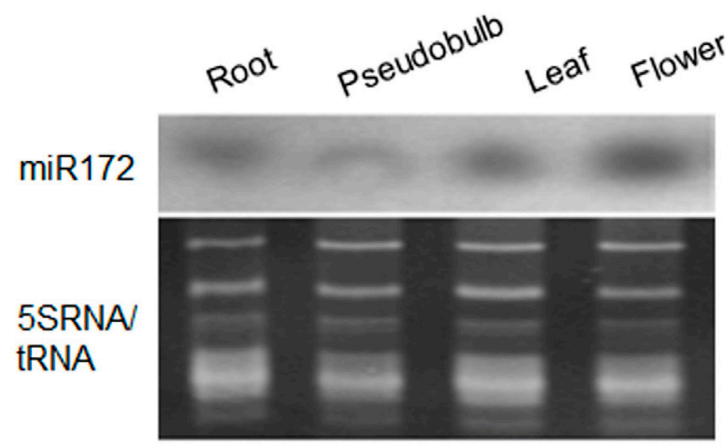

Figure 4. Expression pattern of miR172 in Cymbidium ensifolium. Expression pattern of miR172 in different tissues of $C$. ensifolium by northern blot. Each lane contained $15 \mathrm{mg}$ total RNA, isolated from roots, pseudobulbs, leaves, and flowers. Ethidium bromide staining of 5S RNA/tRNA is shown at the bottom as a loading control.

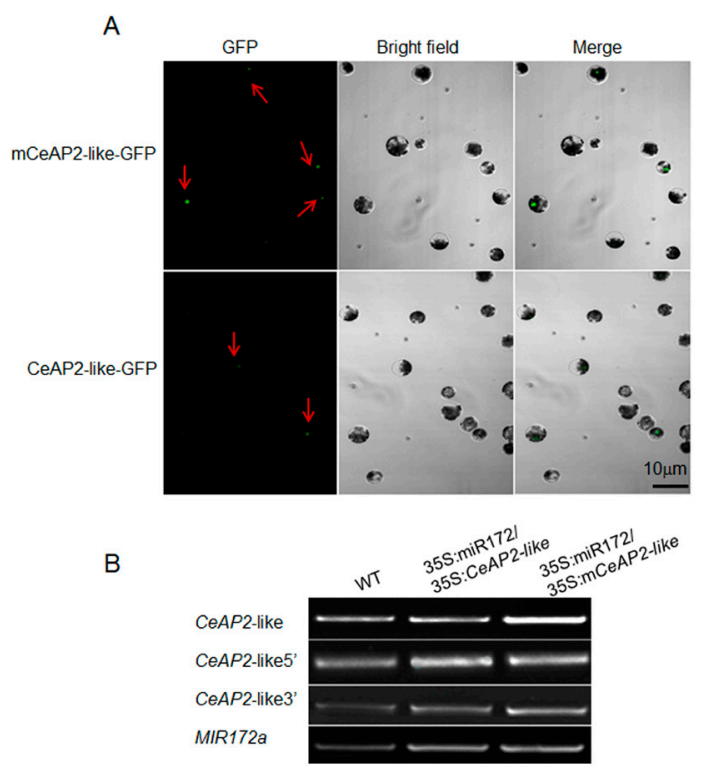

Figure 5. Negative regulation of CeAP2-like expression by miR172. A. 35S:miR172/35S:CeAP2-like and 35S:miR172/35S:mCeAP2-like were co-transformed into Arabidopsis leaf protoplasts by a PEG-mediated method. Signal of fusion protein was visualized by fluorescence microscopy. Bar $=10 \mu \mathrm{m}$. B. Relative expression levels of CeAP2-like in Arabidopsis leaf protoplasts coexpressing 35S:miR172/35S:CeAP2-like and 35S:miR172/35S:mCeAP2-like. MIR172a was used as the internal control. Top lane: full-length of CeAP2-like; 2nd lane: 5 'cleavage fragment, 3 rd lane: 3 'cleavage fragment.

\section{Expression pattern of CeAP2-like in various floral organs}

In light of the differential expression of CeAP2-like shown by mRNA differential display experiment, we reexamined its expression pattern in wild-type and multitepal flowers. Using the northern blotting technique, strong expression signals were detected throughout different floral developmental stages in the wild type (Figure 6A). In contrast, CeAP2-like 
transcript levels appeared to be low or absent after stage 4 in mutant flowers (Figure 6B). The accumulation of CeAP2-like mRNA was also determined in different floral organs (Figure 7). The highest levels of CeAP2-like transcripts in wild-type plants were found in sepals and then in the petals and the gynostemium, which was consistent with the finding in Arabidopsis that AP2 mRNA accumulates predominantly in the outer floral whorls (Wollmann et al., 2010). However, the situation was different in the mutant flower that exhibited petal-to-sepal transformations and failed to generate normal petals. The overall expression level of CeAP2like was lower in the mutant than in the wild type. As indicated by northern blotting shown in Figure 7 (top lane), almost 2 times more total RNA was loaded to obtain a comparable band of CeAP2-like in the mutant compared to that in the wild-type plant. In addition, CeAP2-like mRNA amount was greatly decreased in the central part of the mutant flower, likely due to the absence of a gynostemium in the center. These results revealed that CeAP2-like expression was modified in the multitepal flowers, which further suggested an important role of CeAP2like in the floral development of C. ensifolium.

A

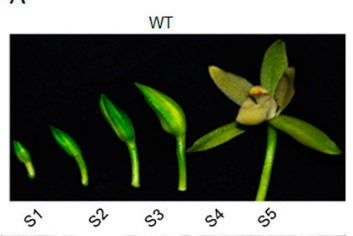

Ce-AP2like

IRNA

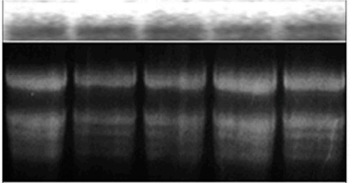

B

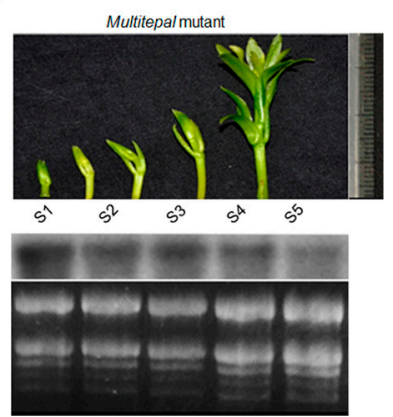

Figure 6. Expression pattern of CeAP2-like in different floral organs. Total RNA was isolated from flowers of wild type and the mutiltepal mutant from stage 1 to stage 5. Each lane contains $15 \mathrm{mg}$ total RNA for the wild type, and $25 \mathrm{mg}$ total RNA for the mutant, normalized to 5S RNA/tRNA. Stage $1<0.5 \mathrm{~cm}$; Stage 2, 0.5 to $1.0 \mathrm{~cm}$; Stage 3, 1.0 to $2.0 \mathrm{~cm}$; Stage 4, 2.0 to $3.0 \mathrm{~cm}$; Stage 5, mature flower.

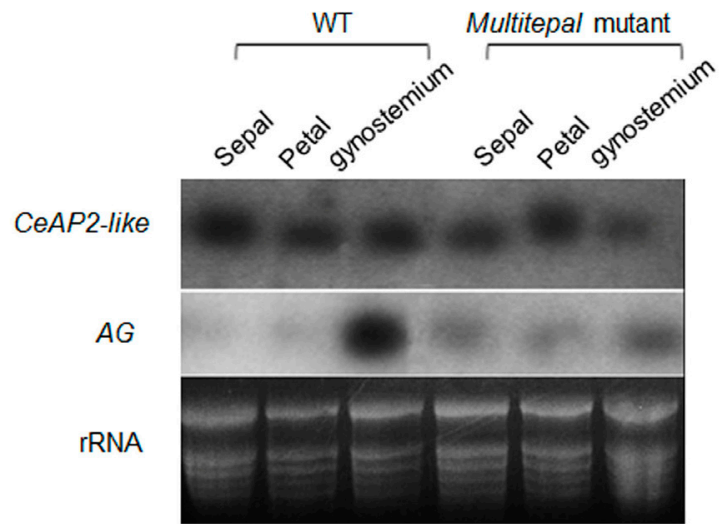

Figure 7. Mutual antagonism between CeAP2-like and $A G$. Expression pattern of CeAP2-like and $A G$ in different floral organs. Each lane contains $15 \mathrm{mg}$ total RNA for the wild type and $25 \mathrm{mg}$ total RNA for the mutant, normalized to 5S RNA/tRNA. 


\section{Involvement of the mutual antagonism between $C e A P 2$-like and $A G$ in the stamen} and petal identity of $C$. ensifolium

In addition to specifying petal identity, $A P 2$ has also been shown to function as an antagonistic gene of $A G$, repressing its expression in floral outer whorls (Wollmann et al., 2010). We therefore compared the transcript dynamics of CeAP2-like and $A G$ in wild-type and multitepal flowers to determine the potential function of CeAP2-like during floral patterning regulation in C. ensifolium. CeAP2-like transcript abundance was dominant in the sepals and was lower in the petals and the gynostemium of wild-type flowers (Figure 7). In comparison, the transcript levels of $A G$ were abundant in the gynostemium and very low in the sepals. This contrasting distribution pattern was similar to that observed in Arabidopsis (Wollmann et al., 2010), and indicated that a mutual antagonism existed between CeAP2-like and $A G$ in $C$. ensifolium. However, this antagonistic distribution was altered in mutant flowers, and a comparable expression of $A G$ was observed in different floral organs, rather than the predominant expression in the center of the flower found in wild type. This phenomenon was probably due to the absence of a gynostemium in the mutant, which prevented the accumulation of $A G$ transcripts in the floral center. Taken together, these results indicate that the antagonism between $A P 2$ and $A G$ is conserved in the orchid plant and that the CeAP2-like/AG regulatory node is likely to act in the regulation of floral patterning of $C$. ensifolium.

\section{DISSCUSSION}

We performed mRNA differential display experiments in wild-type and multitepal flowers of Cymbidium, with the aim of identifying the genes responsible for their differences in floral traits and development. Most sequences were up-regulated in the mutant, however, an AP2-like gene was expressed to a lesser degree in the mature multitepal flower. Further analysis revealed that the isolated CeAP2-like cDNA was very closely related to $A P 2-9$ from E. pusilla. Relatively high degrees of homology were also indicated between this cDNA and the AP2-like sequences from Arabidopsis. Additionally, we showed strong complementarity between CeAP2-like mRNA and miR172, as well as localization of the complementary nucleotides similar to that in the cDNAs of other homologous genes identified in different plant species (Nilsson et al., 2007; Varkonyi-Gasic et al., 2012). These results indicated the potential regulation of $A P 2$ by miR 172 in the orchid.

MicroRNAs (miRNAs) are regulatory small RNAs that repress the expression of their target genes via mRNA cleavage or translational repression (Chiba and Green, 2009; Huntzinger and Izaurralde, 2011). Hundreds of miRNA families have been identified as playing crucial roles in many developmental and physiological processes in different plant species (Jones-Rhoades and Bartel, 2004; Zhang et al., 2006). However, despite reports on small RNA sequencing in orchid, no previous studies have investigated the function of miRNAs in orchid species (An et al., 2011; Lin et al., 2013).

One of the most ancient miRNAs in plants is miR172, and it regulates a small group of AP2 domain-containing, plant-specific transcription factors (Wollmann et al., 2010). Recent studies in both dicotyledons and monocotyledons have shown that miR172 is important in regulating phase transition and determining floral organ identity, although it causes differing morphological outcomes in different species (Glazińska et al., 2009; Zhu et al., 2011). In the present study, we detected the expression of this miRNA in floral organs using an miR172a 
probe. Our results indicated that miR172 was broadly expressed throughout different developmental stages and showed an increased accumulation in the floral organs, which implied that miR172 plays a conserved role in the vegetative development of $C$. ensifolium. Moreover, the decreases in both the fluorescence signals, and the $A P 2$ transcripts of Arabidopsis protoplasts transiently coexpressing miR172/CeAP2-like-GFP compared to those expressing miR172resistant $m C e A P 2$-like-GFP, indicated a negative regulatory role of miR172 on CeAP2-like. Therefore, we assumed that the identified sequence was the cDNA of the miR172-targeted $A P 2$ gene in $C$. ensifolium. These investigations demonstrated that the interaction between miR172 and its $A P 2$ or $A P 2$-like targets is deeply conserved and that the miR172-AP2 family interaction has long been a critical component of plant development.

The role of $A P 2$ in specifying floral organ identity has been widely reported (Chung et al., 2010). Detailed analysis in Arabidopsis has confirmed the mutual antagonism between $A P 2$ and $A G$, leading to the development of a model in which $A P 2$ activity restricts the $A G$ expression domain to determine whether stamens or petals develop (Wollmann et al., 2010). In the present study, the highest accumulation of CeAP2-like was observed in the sepalsof wild-type flowers. Conversely, the transcripts of $A G$ were most prevalent in the gynostemium and least prevalent in the sepals, indicating an antagonistic relationship between CeAP2-like and $A G$, as has been shown previously in Arabidopsis. However, the expression pattern of CeAP2-like/AG was significantly different in the mutant from that in the wild type. Northern blotting analysis indicated lower transcript abundances of $A G$ in the gynostemiums, as well as lower expression level of CeAP2-like in the sepals and across the whole flowers of the mutants, compared to those of the wild-type plants. Despite the correspondence between the reduced CeAP2-like mRNA and the petals in mutant flowers, this result was also likely due to the absence of a gynostemium in the floral center of the mutant, which further reduced levels of $A G$ and its antagonism to $A P 2$ expression. Thus, the $\mathrm{Ce} A P 2$-like/AG regulatory node is likely to act in the regulation of floral patterning. However, additional physiological and molecular data are needed to confirm this hypothesis. Further studies on the overexpression and/ or RNAi of CeAP2-like, together with physiological experiments using transgenic plants, will help explain the function of this gene in the regulation of floral patterning in C. ensifolium.

\section{Conflicts of interest}

The authors declare no conflict of interest.

\section{ACKNOWLEDGMENTS}

Research supported by grants from the Strategic Emerging Industries of Guangdong Province (\#2012A020800003) and grants from the National Natural Science Foundation of China (\#31471915).

\section{Supplementary material}

\section{REFERENCES}

Abel S and Theologis A (1994). Transient transformation of Arabidopsis leaf protoplasts: a versatile experimental system to study gene expression. Plant J. 5: 421-427. 
An FM, Hsiao SR and Chan MT (2011). Sequencing-based approaches reveal low ambient temperature-responsive and tissue-specific microRNAs in Phalaenopsis orchid. PLoS One 6: e18937.

Bowman JL, Smyth DR and Meyerowitz EM (1989). Genes directing flower development in Arabidopsis. Plant Cell 1: 37-52.

Bowman JL, Smyth DR and Meyerowitz EM (1991). Genetic interactions among floral homeotic genes of Arabidopsis. Development 112: 1-20.

Causier B, Schwarz-Sommer Z and Davies B (2010). Floral organ identity: 20 years of ABCs. Semin. Cell Dev. Biol. 21: 73-79.

Chang YY, Kao NH, Li JY, Hsu WH, et al. (2010). Characterization of the possible roles for B class MADS box genes in regulation of perianth formation in orchid. Plant Physiol. 152: 837-853.

Chiba Y and Green PJ (2009). mRNA degradation machinery in plants. J. Plant Biol. 52: 114-124.

Chung MY, Vrebalov J, Alba R, Lee J, et al. (2010). A tomato (Solanum lycopersicum) APETALA2/ERF gene, SlAP2a, is a negative regulator of fruit ripening. Plant J. 64: 936-947.

Dressler RL (1993). Phylogeny and classification of the orchid family. Cambridge University Press, Cambridge, 124-136.

Dressler RL (2005). How many orchid species? Selbyana 26: 155-158.

Eveland AL, Goldshmidt A, Pautler M, Morohashi K, et al. (2014). Regulatory modules controlling maize inflorescence architecture. Genome Res. 24: 431-443.

Ghazoul J (2006). Floral diversity and the facilitation of pollination. J. Ecol. 94: 295-304.

Glazińska P, Zienkiewicz A, Wojciechowski W and Kopcewicz J (2009). The putative miR172 target gene InAPETALA2like is involved in the photoperiodic flower induction of Ipomoea nil. J. Plant Physiol. 166: 1801-1813.

Hsiao YY, Huang TH, Fu CH, Huang SC, et al. (2013). Transcriptomic analysis of floral organs from Phalaenopsis orchid by using oligonucleotide microarray. Gene 518: 91-100.

Huang WT, Fang ZM, Zeng SJ, Zhang JX, et al. (2012). Molecular cloning and functional analysis of three FLOWERING LOCUS T (FT) homologous genes from chinese cymbidium. Int. J. Mol. Sci. 13: 11385-11398.

Huntzinger E and Izaurralde E (2011). Gene silencing by microRNAs: contributions of translational repression and mRNA decay. Nat. Rev. Genet. 12: 99-110.

Jones-Rhoades MW and Bartel DP (2004). Computational identification of plant microRNAs and their targets, including a stress-induced miRNA. Mol. Cell 14: 787-799.

Keck E, McSteen P, Carpenter R and Coen E (2003). Separation of genetic functions controlling organ identity in flowers. Embo J. 22: 1058-1066.

Liang G, He H, Li Y, Wang F, et al. (2014). Molecular mechanism of microRNA396 mediating pistil development in Arabidopsis. Plant Physiol. 164: 249-258.

Lin CS, Chen JJ, Huang YT, Hsu CT, et al. (2013). Catalog of Erycina pusilla miRNA and categorization of reproductive phase-related miRNAs and their target gene families. Plant Mol. Biol. 82: 193-204.

Melzer R and Theissen G (2009). Reconstitution of 'floral quartets' in vitro involving class B and class E floral homeotic proteins. Nucleic Acids Res. 37: 2723-2736.

Melzer R, Verelst W and Theissen G (2009). The class E floral homeotic protein SEPALLATA3 is sufficient to loop DNA in 'floral quartet'-like complexes in vitro. Nucleic Acids Res. 37: 144-157.

Nilsson L, Carlsbecker A, Sundas-Larsson A and Vahala T (2007). APETALA2 like genes from Picea abies show functional similarities to their Arabidopsis homologues. Planta 225: 589-602.

Ó’Maoiléidigh DS, Graciet E and Wellmer F (2014). Gene networks controlling Arabidopsis thaliana flower development. New Phytol. 201: 16-30.

Pelaz S, Ditta GS, Baumann E, Wisman E, et al. (2000). B and C floral organ identity functions require SEPALLATA MADS-box genes. Nature 405: 200-203.

Schiestl FP and Cozzolino S (2008). Evolution of sexual mimicry in the orchid subtribe orchidinae: the role of preadaptations in the attraction of male bees as pollinators. BMC Evol. Biol. 8: 27.

Soltis PS and Soltis DE (2014). Flower diversity and angiosperm diversification. Methods Mol. Biol. 1110: 85-102.

Su CL, Chen WC, Lee AY, Chen CY, et al. (2013). A modified ABCDE model of flowering in orchids based on gene expression profiling studies of the moth orchid Phalaenopsis aphrodite. PLoS One 8: e80462.

Teo ZW, Song S, Wang YQ, Liu J, et al. (2014). New insights into the regulation of inflorescence architecture. Trends Plant Sci. 19: 158-165.

Theissen G and Becker A (2004). Gymnosperm orthologues of class B floral homeotic genes and their impact on understanding flower origin. Crit. Rev. Plant Sci. 23: 129-148.

Tsai WC and Chen HH (2006). The orchid MADS-box genes controlling floral morphogenesis. Sci. World J. 6: 1933-1944.

Tsai WC, Pan ZJ, Hsiao YY, Jeng MF, et al. (2008). Interactions of B-class complex proteins involved in tepal development in Phalaenopsis orchid. Plant Cell Physiol. 49: 814-824.

Varkonyi-Gasic E, Lough RH, Moss SM, Wu R, et al. (2012). Kiwifruit floral gene APETALA2 is alternatively spliced and accumulates in aberrant indeterminate flowers in the absence of miR172. Plant Mol. Biol. 78: 417-429. 
Wang L, Albert NW, Zhang H, Arathoon S, et al. (2014). Temporal and spatial regulation of anthocyanin biosynthesis provide diverse flower colour intensities and patterning in Cymbidium orchid. Planta 240: 983-1002.

Wang SY, Lee PF, Lee YI, Hsiao YY, et al. (2011). Duplicated C-class MADS-box genes reveal distinct roles in gynostemium development in Cymbidium ensifolium (Orchidaceae). Plant Cell Physiol. 52: 563-577.

Wollmann H, Mica E, Todesco M, Long JA, et al. (2010). On reconciling the interactions between APETALA2, miR172 and AGAMOUS with the ABC model of flower development. Development 137: 3633-3642.

Yang F, Liang G, Liu D and Yu D (2009). Arabidopsis miR396 mediates the development of leaves and flowers in transgenic tobacco. J. Plant Biol. 52: 475-481.

Yang YX, Wang JJ, Ma ZH, Sun GS, et al. (2014). De novo sequencing and comparative transcriptome analysis of white petals and red labella in Phalaenopsis for discovery of genes related to flower color and floral differentation. Acta Soc. Bot. Pol. 83: 191-199.

Zhang B, Pan X, Cannon CH, Cobb GP, et al. (2006). Conservation and divergence of plant microRNA genes. Plant J. 46: 243-259.

Zhu QH and Helliwell CA (2011). Regulation of flowering time and floral patterning by miR172. J. Exp. Bot. 62: 487-495. 